\title{
Rational design of light-controlled microrobots
}

Bunea, Ada-loana; Engay, Einstom; Chouliara, Manto; Bañas, Andrew Rafael; Glückstad, Jesper

\section{Published in:}

Proceedings of SPIE

Link to article, DOI:

$10.1117 / 12.2325547$

Publication date:

2018

Document Version

Publisher's PDF, also known as Version of record

Link back to DTU Orbit

Citation (APA):

Bunea, A-I., Engay, E., Chouliara, M., Bañas, A. R., \& Glückstad, J. (2018). Rational design of light-controlled microrobots. In Proceedings of SPIE (Vol. 10804 ). [1080406] SPIE - International Society for Optical Engineering. Proceedings of SPIE - The International Society for Optical Engineering https://doi.org/10.1117/12.2325547

\section{General rights}

Copyright and moral rights for the publications made accessible in the public portal are retained by the authors and/or other copyright owners and it is a condition of accessing publications that users recognise and abide by the legal requirements associated with these rights.

- Users may download and print one copy of any publication from the public portal for the purpose of private study or research.

- You may not further distribute the material or use it for any profit-making activity or commercial gain

- You may freely distribute the URL identifying the publication in the public portal 


\section{Rational design of light-controlled microrobots}

\section{Ada-Ioana Bunea, Einstom Engay, Manto Chouliara, Andrew Rafael Bañas, Jesper Glückstad}

Ada-loana Bunea, Einstom Engay, Manto Chouliara, Andrew Rafael Bañas, Jesper Glückstad, "Rational design of light-controlled microrobots," Proc. SPIE 10804, Advanced Manufacturing Technologies for Micro- and Nanosystems in Security and Defence, 1080406 (8 October 2018); doi: $10.1117 / 12.2325547$

SPIE. Event: SPIE Security + Defence, 2018, Berlin, Germany 


\title{
Rational design of light-controllable microrobots
}

\author{
Ada-Ioana Bunea*, Einstom L. Engay, Manto Chouliara, Andrew R. Bañas, Jesper Glückstad \\ DTU Fotonik, Dept. of Photonics Engineering, Technical University of Denmark, \\ DK-2800, Kgs. Lyngby, Denmark
}

\begin{abstract}
Light Robotics is one of the newest progenies of the robotics family, bringing together advances in microfabrication and optical manipulation with intelligent control ideas from robotics and Fourier optics. The development of lightcontrollable microrobots capable of performing specific tasks at the microscale requires the ability to sculpt the two protagonists of the story: the light and the microrobots. Complex light sculpting for optical trapping has been in focus for over three decades, and its importance for controlling microscopic objects is well understood. Designing intricate microrobots for the task is a more recent development facilitated by state-of-the-art microfabrication techniques, and particularly by two-photon polymerization. The full 3D design freedom offered by two-photon polymerization opens the door for imagination, while at the same time bringing the responsibility of rationally designing microrobots tailored to specific tasks. In addition to shape and topology features, the surface chemistry of the microrobots can also help steer them towards specific applications. This paper will discuss strategies for the design and fabrication of light-controllable microrobots as a toolbox for biomedical applications.
\end{abstract}

Keywords: Light Robotics, microrobots, two-photon polymerization, optical manipulation, GPC, surface modification

\section{INTRODUCTION}

Recent technological advancements in micro-and nanofabrication have facilitated the development of micro- and nanorobots with various designs, control mechanisms and purposes. Despite existing technological challenges, mobile micro- and nanorobots have already shown great potential for a wide range of applications, especially in the biomedical field $^{1-3}$. However, the gap between proof-of-concept laboratory experiments and real-life applications is rather large. Thus, further testing and optimization is required before micro- and nanorobots could become able to carry out a variety of tasks at the microscale and particularly in the human body. Rational design of the next generation of micro- and nanorobots in relation to a specific application should help further progress in the field at an accelerated rate.

\subsection{Control modalities for microrobots}

The motion of micro- and nanorobots can be induced with the aid of microorganisms ${ }^{4,5}$, or through several physical and chemical mechanisms, which give the user variable degrees of freedom for their control.

Self-propulsion of small objects fueled by the catalytic decomposition of hydrogen peroxide was first reported in $2002^{6}$. This lead to the development of different chemically-propelled micro- and nanomotors ${ }^{7-9}$ that move through mechanisms such as bubble propulsion, self-electrophoresis or self-diffusiophoresis. Since hydrogen peroxide is a rather toxic compound, alternative fuel sources have been investigated, and enzyme-based nanomotors seem to show the most promise $^{10}$. Chemically propelled micro-and nanomotors have been employed for different biomedical and environmental applications $^{11}$ such as biosensing ${ }^{12}$, water purification ${ }^{13}$ or as synthetic repair systems ${ }^{14}$. However, these chemically powered microrobots are autonomous rather than controllable by a user.

In addition to bacteria-aided and chemical propulsion, several physical control mechanisms have been employed for micro- and nanorobots, such as magnetic ${ }^{15,16}$, electrostatic $^{17,18}$, thermal ${ }^{19,20}$ and optical ${ }^{21,22}$. Physical control mechanisms offer rather precise control over the motion of the microscopic tools. Figure 1 shows a schematic representation of the most commonly used control modalities for micro- and nanorobots.

Advanced Manufacturing Technologies for Micro- and Nanosystems in Security and Defence, edited by

Andrea Camposeo, Yuris Dzenis, Maria Farsari, Luana Persano, Proc. of SPIE Vol. 10804, 1080406

(C) 2018 SPIE · CCC code: $0277-786 X / 18 / \$ 18 \cdot$ doi: $10.1117 / 12.2325547$ 


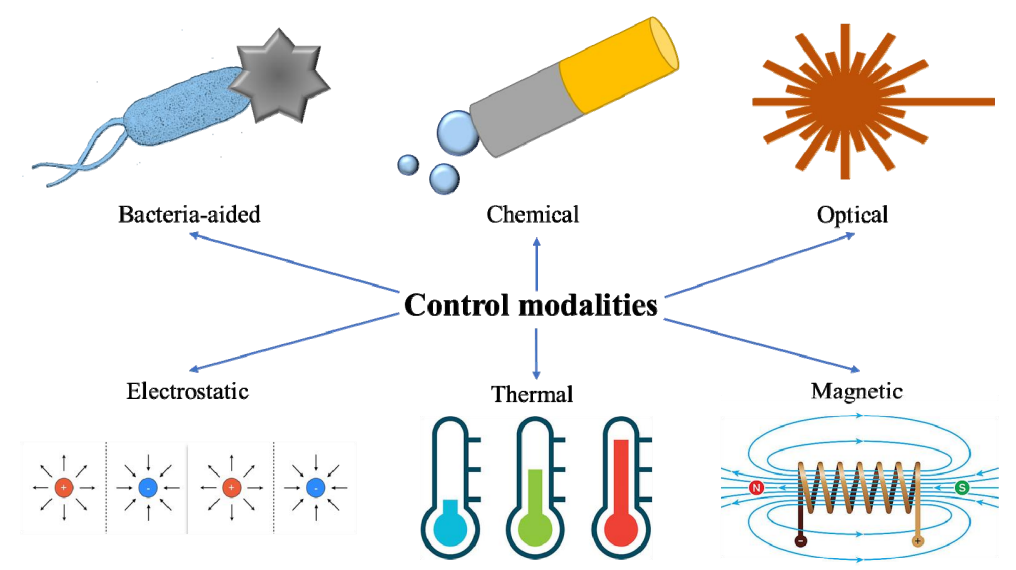

Figure 1. Most common motion-inducing control modalities for micro- and nanorobots.

\subsection{Light as the actuator for microrobots}

The use of light as actuator comes with its advantages and disadvantages. Energy transfer between the light source and the microrobots is done without physical contact. Additionally, light can travel over extended distances in the absence of absorbent or scattering materials. Thus, light actuation is minimally invasive for both the microrobots and their surroundings, which is particularly interesting for biomedical applications. However, when it comes to using light as actuator in biological samples, things become complicated due to the optical properties of the environment. Advanced light-shaping methods might be able to overcome the challenges of optical actuation in biological samples ${ }^{3}$.

The properties of light, such as energy, polarization, pulse duration or illumination direction, can be adjusted in real time and enable the motion control or triggering specific functions of the microrobots ${ }^{22}$. Different methods, both direct and indirect, can be employed for actuating microrobots using light. Phototactic control of microstructures with the aid of flagellated bacteria was achieved using UV light ${ }^{23}$. Alternatively, light-responsive materials, such as liquid crystalline polymers, hydrogels or elastomers, can be used for fabricating microrobots, which then exhibit specific responses to light stimuli through e.g. shape changes ${ }^{22}$. Direct control of microrobots using light can also be achieved through optical trapping using highly-focused laser beams ${ }^{21}$.

\subsection{Optical trapping and manipulation using Generalized Phase Contrast}

Generalized Phase Contrast (GPC) is a photon-efficient phase-only light shaping technique ${ }^{24,25}$. GPC-based light shaping allows simultaneous optical trapping and 3D-manipulation of up to tens of particles ${ }^{26}$. Compared to standard optical tweezers, which make use of high numerical aperture (NA) objectives, GPC makes use of low NA objectives. This increases the operating volume for optical trapping and manipulation and facilitates the incorporation of side-view into the system, which we employ in our Biophotonics Workstation ${ }^{27}$. Combining top- and side-view imaging facilitates better monitoring and control of the microrobot motion and therefore more precise actuation.

This paper will focus on discussing strategies for the design, fabrication and functionalization of 3D-printed microrobots that allow optical trapping and manipulation.

*adabu@fotonik.dtu.dk; phone +45 452566 33; www.ppo.dk 


\section{TWO-PHOTON POLYMERIZATION AS RAPID PROTOTYPING TECHNIQUE}

\subsection{Rapid prototyping techniques}

In the past three decades, microfabrication techniques have experienced rapid growth due to the increasing need for miniaturized components in IT, biomedicine, transport industry or telecommunications ${ }^{28}$. Among 3D micro-additive manufacturing techniques, several classifications exist, according to e.g. the method employed, the process "dimension", or the materials ${ }^{29}$.

Rapid prototyping techniques rely on transforming data from computer-aided design (CAD) drawings into 3D objects that reproduce the $3 \mathrm{D}$ design ${ }^{30}$. This is achieved by first digitally slicing the $3 \mathrm{D}$ design, followed by the transformation of each 2D layer into a voxel matrix and layer-by-layer fabrication. Stereolithography was developed in the early $1980 \mathrm{~s}^{31,32}$ and became the first commercially available rapid prototyping technique in 1986, from the 3D Systems Corporation $^{33}$. Although rapid prototyping techniques were established mainly for product development, their use has recently extended to rapid manufacturing of small series of products at industrial level.

Classic rapid prototyping techniques have a maximum resolution of several micrometers ${ }^{34}$. The only rapid prototyping technique that is able to achieve a resolution beyond the diffraction limit is two-photon polymerization (2PP), which enables the fabrication of features as small as $100 \mathrm{~nm}^{34-37}$. $2 \mathrm{PP}$ is the highest resolution available rapid prototyping technique, but this comes at the price of increased fabrication times ${ }^{38}$.

\subsection{Two-photon polymerization: the basics}

In 2PP, a near-infrared (NIR) highly-focused femtosecond-pulsed laser beam is scanned with high spatial accuracy in a liquid photoresist transparent in NIR which contains two-photon absorption (2PA) chromophores and suitable photoinitiators $^{36}$. Excitation of the 2PA chromophores makes them emit fluorescent light in the UV-Vis range, which leads to the excitation of the photoinitiator and the generation of radicals that further react with nearby monomers and oligomers, leading to their crosslinking ${ }^{36}$.

The laser beam is scanned in the xy-plane using either the raster scanning or the contour scanning method to generate a solid photoresist layer. Subsequently, the following layers are fabricated in a similar manner after translating the beam focus along the z-axis with the aid of the piezoelectric stage. After printing, the 3D structures obtained are developed in a suitable solvent, which selectively removes the uncrosslinked photoresist. A schematic of 2PP is shown in Figure 2.

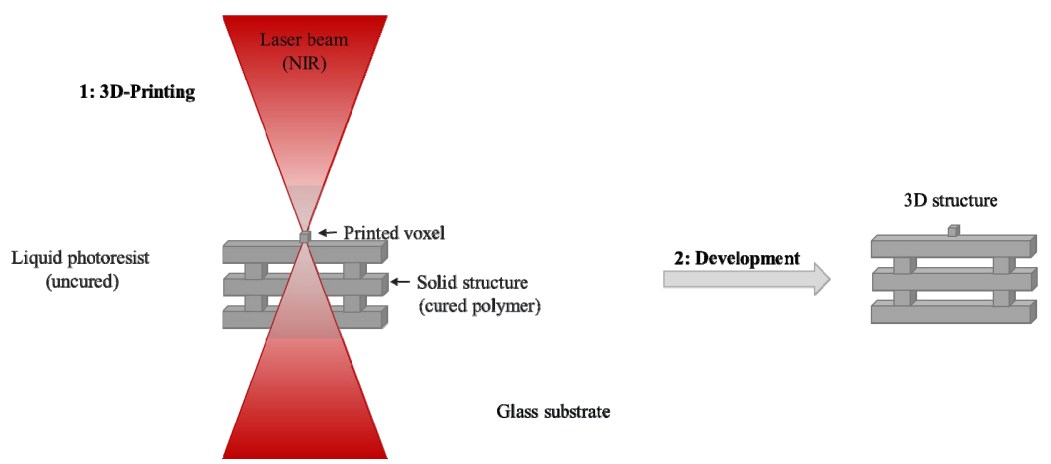

Figure 2. Schematic representation of 3D printing using two-photon polymerization.

For maximizing resolution while maintaining the integrity of the 3D printed structures, the printing parameters need to be optimized. The choice of monomer, photoinitiator and 2PA chromophore influences the maximum resolution achievable. For a given photoresist formulation, the laser power, the slicing and hatching distances and the stage translation parameters will determine the resolution of the printed structures. 


\subsection{Two-photon polymerization in our laboratory}

Using a rapid prototyping technique facilitates fast design and fabrication iterations, which is essential for optimizing the design of our microrobots. We employ a Nanoscribe Photonic Professional GT system (Nanoscribe GmbH, Germany) for 2PP-based 3D-printing. As polymer precursor, we use Nanoscribe IPL-80, a commercial acrylic-based photoresist.

For printing, we fix the power scaling to correspond to $16 \mathrm{~mW}$ average power at the aperture objective and we set the slicing and hatching distances to $200 \mathrm{~nm}$ and the stage velocity to $200 \mu \mathrm{m} \cdot \mathrm{s}^{-1}$ (optimized values). After printing, we develop the structures by immersing the substrate in isopropanol for $20 \mathrm{~min}$ to ensure the removal of uncrosslinked photoresist. With these parameters, we achieve a resolution of approximately $200 \mathrm{~nm}$, as determined from scanning electron microscopy (SEM) images (Figure 3), in good agreement with the producer specifications for the system.

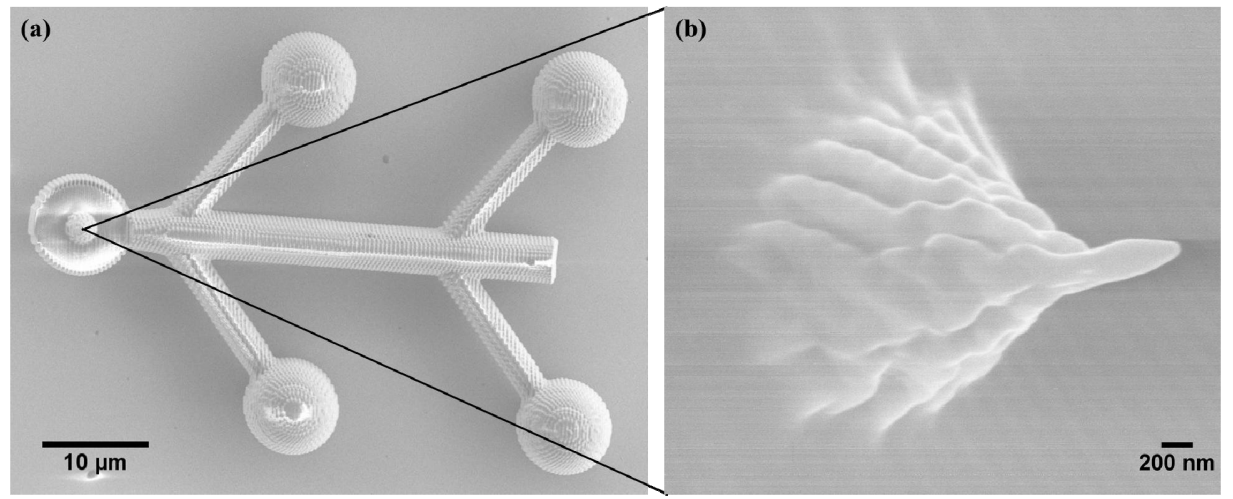

Figure 3. Scanning electron microscopy image of a disk-tip microrobot fabricated using two-photon polymerization. (b) shows a zoom-in of the tip feature. The print lines are clearly visible and thus allow an estimation of the printing resolution.

\section{DESIGN OF LIGHT-CONTROLLABLE MICROROBOTS}

\subsection{General considerations}

Sculpting the object is of particular importance for developing microrobots tailored to specific tasks at the microscale ${ }^{39}$. Including spherical "wheels" or "handles" as part of the microrobot design facilitates optical trapping in the Mie regime when the size of the trapped spheres is significantly larger than the wavelength of the trapping beam ${ }^{40}$. The size and number of such "handles" are two of the design parameters to be taken into account in the design. In addition to the "handles", different features can be included into the microrobot design to enable the microbot to perform a variety of tasks at the microscale. Figure 4 shows examples of microrobot designs that allow optical trapping and manipulation.

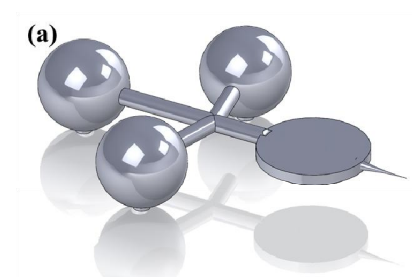

(c)

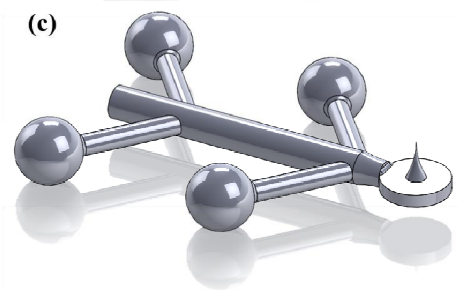

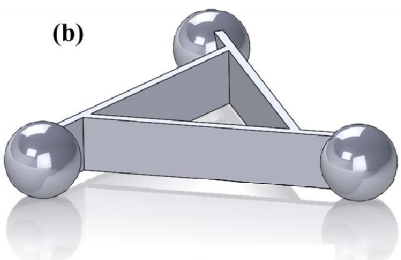

(d)

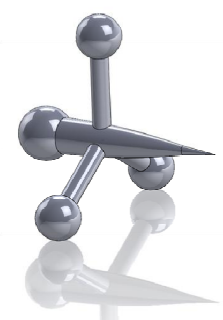

Figure 4. Sample designs of light-controllable microrobots. (a) was employed for generating a natural convection flow in a closed microfluidic channel ${ }^{41}$, while (b) was used as support structure for maneuvering polystyrene microspheres ${ }^{42}$. 


\subsection{Microrobot designs from our laboratory}

Our research group has been working on multiple microrobot designs for different applications ${ }^{43}$. For example, including a relatively large disk as part of the design can allow the microrobot to push and align microscopic structures that cannot be directly manipulated using light. With the aid of off-resonant plasmonic heating of a gold-coated disk, micromixing can be enhanced in closed microchannels ${ }^{41}$. Including a sharp tip as part of the microrobot can facilitate local mechanical perturbation of biological samples such as mucus, lipid bilayers or even cell membranes, which should enable bursting open a bacteria cell or perforating plant cell walls. By designing a hollow microrobot that can be heated we introduced a syringe function for suctioning small particles from its immediate proximity and delivering the particles to a different location $^{21}$. We have also reported wave-guided optical waveguides that have potential as single-cell tip-enhanced Raman scattering probes ${ }^{44,45}$. All these applications were made possible by the rational design of the microtools in relation to the target application. Countless other tasks at the microscale can be identified, and specialized microrobots can be tailored to these tasks. Together, these microrobot designs form a rapidly-growing toolbox for biomedical applications which might help shape the future of research.

\section{SURFACE FUNCTIONALIZATION OF THE MICROROBOTS}

The surface chemistry properties of the microrobots can be tailored in various ways to add extra functionality. The polymer precursor determines the surface chemistry immediately after printing. Additional fabrication steps can be included in order to modify either the entire surface or target areas of the microrobots. The following subsections will discuss some of the possible approaches to fabricate microrobots with specific surface properties.

\subsection{The choice of photoresist}

A with range of both positive and negative photoresists can be employed for $2 \mathrm{PP}$, with negative photoresists being the most common ${ }^{46,47}$. Acrylic-based photoresists developed in-house ${ }^{48}$ or commercially-available ${ }^{49}$, as well as SU- ${ }^{47}$, an epoxy-based negative photoresist, are widely used in photolithography, including 2PP. Various other biocompatible materials, such as Ormocer ${ }^{\circledR}$, metal-sol-gel composites, bovine serum albumin, collagen, gelatin, fibrinogen and biodegradable polycaprolactone-based or polyethylene glycol polymers, have been successfully $3 \mathrm{D}$-printed using $2 \mathrm{PP}^{50}$.

The choice of photoresist influences the maximum resolution of the fabrication process ${ }^{51}$ and the mechanical properties of the obtained structures ${ }^{46}$, while also determining the chemical surface properties of the printed objects, such as wettability and the presence of surface functional groups. The use of biocompatible materials ensures that the printed structures can be employed for biomedical applications.

\subsection{Thin film coatings}

One relatively simple manner to change the surface chemistry of 3D-printed structures is through coating. This can be achieved using a variety of materials via e.g. different thin film deposition techniques. Physical vapor deposition (PVD) and chemical vapor deposition (CVD) techniques are commonly employed to achieve coating thicknesses from atomic level to several tens of micrometers ${ }^{52,53}$. For selective coating of target areas of the microrobots, a mask can be 3Dprinted on top of the microrobot structures to selectively expose the areas of interest for coating ${ }^{21}$.

For improved optical trapping and manipulation, antireflection coatings can be employed to achieve higher trapping forces by reducing the scattering forces ${ }^{54-56}$. This facilitates the stable trapping of high refractive-index structures or the use of lower numerical aperture objectives.

Nanostructured gold coatings can be used to trigger localized heating upon illumination via resonant or off-resonant plasmonic effects ${ }^{41}$. Additionally, including a gold layer as part of the microrobot structure allows further functionalization, which will be discussed in the following subsection.

\subsection{Immobilization of (bio)molecules}

Covalent binding of (bio)molecules on the microrobots' surface can be achieved through chemical reactions between reactive functional groups through well established methods that make use of various coupling reagents ${ }^{57,58}$. Introducing carboxyl or amino functional groups on the microrobots' surface facilitates the immobilization of a wide range of molecules, from relatively small fluorescent dyes to large proteins. Some approaches for surface functionalization are briefly discussed in the following subsections and schematically shown in Figure 5. 
4.3.1. Plasma treatment. Plasma treatment of polymer surfaces can be performed in vacuum, in the presence of pure gases (e.g. oxygen, nitrogen, ammonia, carbon dioxide, water vapor or noble gases) or in the presence of gaseous mixtures (e.g. air) ${ }^{59}$. Depending on the atmosphere, power and duration, plasma treatment can lead to different changes in the treated polymer surfaces ${ }^{60}$. Mild plasma treatment is often used for surface cleaning, as it efficiently removes organic surface contaminants. Stronger plasma treatment is used for surface etching, either to ensure the removal of thin residual photoresist layers or to increase surface roughness. Surface deposition of siloxanes through plasma treatment was also reported ${ }^{61}$.

Plasma treatment leads to surface chemistry and surface energy changes. This facilitates control over the wettability and adhesion properties of the surface. Furthermore, the use of plasma treatment in an atmosphere containing heteroatoms such as nitrogen or oxygen leads to the introduction of functional groups on the surface, such as amino, carboxyl, carbonyl or hydroxyl. However, plasma treatment effects are transitory, so thorough characterization of the surface is essential before performing additional modification steps. X-ray photoelectron spectroscopy (XPS) is the most commonly used technique to investigate plasma treated surfaces and confirm the presence of functional groups.

4.3.2. Photolinker-mediated immobilization. Photoactivatable heterobifunctional crosslinkers (photolinkers) can be employed to attach biomolecules on polymeric surfaces ${ }^{62}$. Due to the light-dependent nature of functionalization with photolinker molecules, a pattern can be defined on the surface in similar manner to photolithographic patterning of polymers $^{63}$. Compared to plasma treatment, the use of photolinkers offers improved control over the density and distribution of functional groups on the surface.

4.3.3. Self-assembly of thiols. Alkanethiols, compounds with the general chemical structure $\mathrm{HS}\left(\mathrm{CH}_{2}\right)_{\mathrm{n}} \mathrm{X}$, can selfassemble from solution onto clean gold surfaces, due to the predilection of the sulfur atom for gold ${ }^{64}$. The self-assembled monolayers (SAMs) formed are densely packed, well ordered, and with the functional group X exposed at the SAM-air or SAM-liquid interface. The properties of the modified surface can be tailored by varying the chain length of the thiol, $\mathrm{n}$, or the functional group $\mathrm{X}^{65}$. To allow further modification of the surface, amino or carboxyl terminal groups are commonly used. Furthermore, mixed SAMs can be generated by using a solution containing a mixture of thiols. Mixed SAMs can facilitate the immobilization of two different molecules in a subsequent surface modification step.

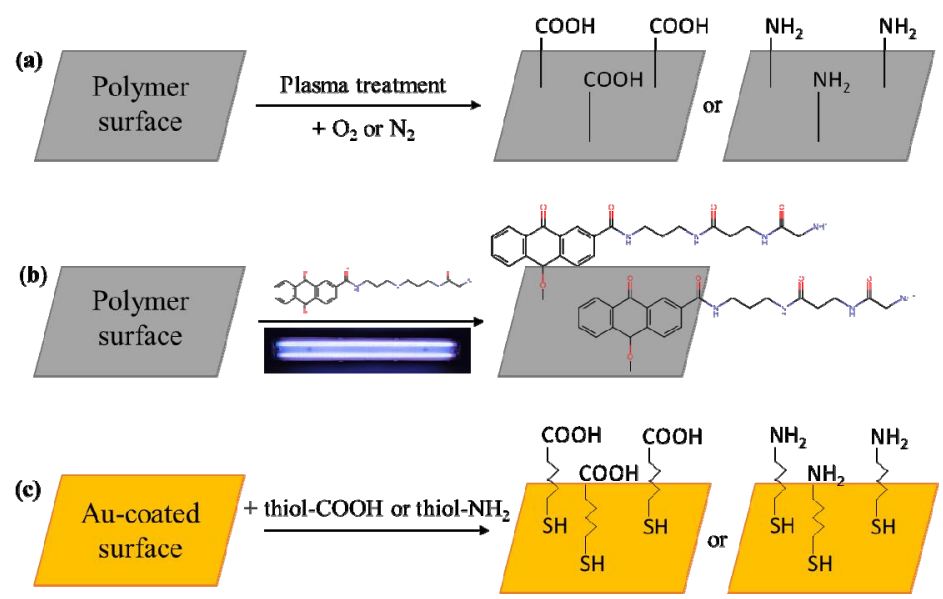

Figure 5. Approaches for introducing functional groups on the microrobot surface: a) plasma treatment; b) UV-assisted grafting of anthraquinone amine photolinkers; c) self-assembly of thiols on gold-coated substrates.

\section{CONCLUSIONS}

Recent advances in the fabrication and actuation of micro- and nanorobots allow them to perform a variety of tasks at the microscale. Among these tools, light-controlled robots are particularly interesting for biomedical research due to the noninvasive nature of light as the actuator. Rational design of light-controllable microrobots for specific tasks requires careful consideration of their shape, size, and surface properties. Combining existing knowledge from microfabrication, polymer chemistry and surface engineering creates limitless design possibilities, which need to be intelligently exploited in order to achieve the best outcome regarding a given task. 
We believe that an interdisciplinary approach combining expertise from micro- and nano-engineering, photonics, chemistry and robotics is needed in order to develop the next generation of light-controlled microrobots and enhance the abilities of such tools.

\section{ACKNOWLEDGEMENTS}

We would like to thank Mogens Havsteen Jakobsen from DTU Nanotech for his help with anthraquinone amine photolinker surface modification.

We acknowledge the Novo Nordisk Foundation (Grand Challenge Program; NNF16OC0021948) for funding this work.

\section{REFERENCES}

[1] Nelson, B. J., Kaliakatsos, I. K. and Abbott, J. J., “Microrobots for Minimally Invasive Medicine,” Annu. Rev. Biomed. Eng. 12(1), 55-85 (2010).

[2] Sitti, M., Ceylan, H., Hu, W., Giltinan, J., Turan, M., Yim, S. and Diller, E., "Biomedical Applications of Untethered Mobile Milli/Microrobots,” Proc. IEEE 103(2), 205-224 (2015).

[3] Glückstad, J. and Palima, D., [Light robotics : structure-mediated nanobiophotonics], Elsevier (2017).

[4] Martel, S., "Bacterial microsystems and microrobots," Biomed. Microdevices 14(6), 1033-1045 (2012).

[5] Behkam, B. and Sitti, M., "Bacterial flagella-based propulsion and on/off motion control of microscale objects," Appl. Phys. Lett. 90(2), 023902 (2007).

[6] Ismagilov, R. F., Schwartz, A., Bowden, N., Whitesides, G. M., Whitesides, G. M., Ismagilov, R. F., Schwartz, A. and Bowden, N., "Autonomous Movement and Self-Assembly," Angew. Chemie 41(4), 652-654 (2002).

[7] Sánchez, S., Soler, L. and Katuri, J., "Chemically Powered Micro- and Nanomotors," Angew. Chemie 54(5), 1414-1444 (2015).

[8] Paxton, W. F., Kistler, K. C., Olmeda, C. C., Sen, A., Angelo, S. K. St., Cao, Y., Mallouk, T. E., Paul E. Lammert and Crespi, V. H., "Catalytic Nanomotors: Autonomous Movement of Striped Nanorods" (2004).

[9] Fournier-Bidoz, S., Arsenault, A. C., Manners, I. and Ozin, G. A., "Synthetic self-propelled nanorotors," Chem. Commun. 0(4), 441 (2005).

[10] Gaspar, S., "Enzymatically induced motion at nano-and micro-scales," Nanoscale 6, 7757-7763 (2014).

[11] Safdar, M., Khan, S. U. and Jänis, J., "Progress toward Catalytic Micro- and Nanomotors for Biomedical and Environmental Applications," Adv. Mater. 30(24), 1703660 (2018).

[12] Bunea, A.-I., Pavel, I.-A., David, S. and Gáspár, S., "Sensing based on the motion of enzyme-modified nanorods," Biosens. Bioelectron. 67, 42-48 (2015).

[13] Vilela, D., Parmar, J., Zeng, Y., Zhao, Y. and Sánchez, S., "Graphene-Based Microbots for Toxic Heavy Metal Removal and Recovery from Water," Nano Lett. 16(4), 2860-2866 (2016).

[14] Li, J., Shklyaev, O. E., Li, T., Liu, W., Shum, H., Rozen, I., Balazs, A. C. and Wang, J., "Self-Propelled Nanomotors Autonomously Seek and Repair Cracks," Nano Lett. 15(10), 7077-7085 (2015).

[15] Kim, S., Qiu, F., Kim, S., Ghanbari, A., Moon, C., Zhang, L., Nelson, B. J. and Choi, H., "Fabrication and Characterization of Magnetic Microrobots for Three-Dimensional Cell Culture and Targeted Transportation," Adv. Mater. 25(41), 5863-5868 (2013).

[16] Diller, E., Giltinan, J. and Sitti, M., "Independent control of multiple magnetic microrobots in three dimensions," Int. J. Rob. Res. 32(5), 614-631 (2013).

[17] Pawashe, C., Floyd, S. and Sitti, M., "Multiple magnetic microrobot control using electrostatic anchoring," Appl. Phys. Lett. 94(16), 164108 (2009).

[18] Donald, B. R., Levey, C. G., Mcgray, C. D., Paprotny, I., Rus, D., Mcgray, C. D. and Paprotny, I., “An Untethered, Electrostatic, Globally Controllable MEMS Micro-Robot,” J. Microelectromechanical Syst. 15(1) (2006).

[19] Erdem, E. Y., Yu-Ming Chen, Mohebbi, M., Suh, J. W., Kovacs, G., Darling, R. B. and Böhringer, K. F., "Thermally Actuated Omnidirectional Walking Microrobot," J. Microelectromechanical Syst. 19(3), 433-442 (2010).

[20] Sul, O. J., Falvo, M. R., Taylor, R. M., Washburn, S. and Superfine, R., "Thermally actuated untethered impactdriven locomotive microdevices," Appl. Phys. Lett. 89(20), 203512 (2006). 
[21] Villangca, M. J., Palima, D., Bañas, A. R. and Glückstad, J., "Light-driven micro-tool equipped with a syringe function," Light Sci. Appl. 5(9), e16148. (2016).

[22] Nocentini, S., Parmeggiani, C., Martella, D. and Wiersma, D. S., “Optically Driven Soft Micro Robotics,” Adv. Opt. Mater., 1800207 (2018).

[23] Steager, E., Kim, C.-B., Patel, J., Bith, S., Naik, C., Reber, L. and Kim, M. J., "Control of microfabricated structures powered by flagellated bacteria using phototaxis," Appl. Phys. Lett. 90(26), 263901 (2007).

[24] Glückstad, J. and Palima, D., [Generalized phase contrast : applications in optics and photonics], Springer (2009).

[25] Bañas, A. and Glückstad, J., "Light Shaping with Holography, GPC and Holo-GPC," Opt. Data Process. Storage 3(1), 20-40 (2017).

[26] Rodrigo, P. J., Daria, V. R. and Glückstad, J., "Real-time three-dimensional optical micromanipulation of multiple particles and living cells.," Opt. Lett. 29(19), 2270-2272 (2004).

[27] Ulriksen, H.-U., Thogersen, J., Keiding, S., Perch-Nielsen, I. R., Dam, J. S., Palima, D. Z., Stapelfeldt, H. and Gluckstad, J., "Independent trapping, manipulation and characterization by an all-optical biophotonics workstation," J. Eur. Opt. Soc. Rapid Publ. 3, 08034 (2008).

[28] Alting, L., Kimura, F., Hansen, H. N. and Bissacco, G., "Micro Engineering," CIRP Ann. 52(2), 635-657 (2003).

[29] Vaezi, M., Seitz, H. and Yang, S., “A review on 3D micro-additive manufacturing technologies," Int. J. Adv. Manuf. Technol. 67(5-8), 1721-1754 (2013).

[30] Peltola, S. M., Melchels, F. P. W., Grijpma, D. W. and Kellomäki, M., "A review of rapid prototyping techniques for tissue engineering purposes," Ann. Med. 40(4), 268-280 (2008).

[31] Kodama, H., "Automatic method for fabricating a three-dimensional plastic model with photo-hardening polymer," Rev. Sci. Instrum. 52(11), 1770-1773 (1981).

[32] Hull, C. W., "Apparatus for production of three-dimensional objects by stereolithography," US4575330A (1986).

[33] Melchels, F. P. W., Feijen, J. and Grijpma, D. W., "A review on stereolithography and its applications in biomedical engineering," Biomaterials 31(24), 6121-6130 (2010).

[34] Farsari, M. and Chichkov, B. N., "Materials processing: Two-photon fabrication," Nat. Photonics 3(8), 450-452 (2009).

[35] Park, S.-H., Yang, D.-Y. and Lee, K.-S., "Two-photon stereolithography for realizing ultraprecise threedimensional nano/microdevices," Laser Photonics Rev. 3(1-2), 1-11 (2009).

[36] Lee, K.-S., Yang, D.-Y., Park, S. H. and Kim, R. H., "Recent developments in the use of two-photon polymerization in precise 2D and 3D microfabrications," Polym. Adv. Technol. 17(2), 72-82 (2006).

[37] Lee, K.-S., Kim, R. H., Yang, D.-Y. and Park, S. H., "Advances in 3D nano/microfabrication using two-photon initiated polymerization," Prog. Polym. Sci. 33(6), 631-681 (2008).

[38] Zhou, X., Hou, Y. and Lin, J., “A review on the processing accuracy of two-photon polymerization,” AIP Adv. 5(3), 030701 (2015).

[39] Glückstad, J., "Optical manipulation: Sculpting the object," Nat. Photonics 5(1), 7-8 (2011).

[40] Villangca, M. J., Casey, D. ; and Glückstad, J., "Optically-controlled platforms for transfection and single-and sub-cellular surgery,” Biophys. Rev. 7(4), 379-390 (2018).

[41] Engay, E., Bunea, A.-I., Chouliara, M., Bañas, A. and Glückstad, J., "Natural convection induced by an optically fabricated and actuated microtool with a thermoplasmonic disk," Opt. Lett. 43(17), (2018).

[42] Bañas, A., Vizsnyiczai, G., Búzás, A., Palima, D., Kelemen, L., Ormos, P. and Glückstad, J., "Fabrication and optical trapping of handling structures for re-configurable microsphere magnifiers," Proc. SPIE 8637, 86370Y (2013).

[43] Palima, D. ; and Glückstad, J., "Gearing up for optical microrobotics: micromanipulation and actuation of synthetic microstructures by optical forces," Laser Photon. Rev. 7(4), 478-494 (2013).

[44] Palima, D., Bañas, A. R., Vizsnyiczai, G., Kelemen, L., Ormos, P. and Glückstad, J., "Wave-guided optical waveguides," Opt. Express 20(3), 2004-2014 (2012).

[45] Villangca, M. J., Bañas, A., Palima, D. and Glückstad, J., "Generalized phase contrast-enhanced diffractive coupling to light-driven microtools," Opt. Eng. 54(11), 111308 (2015).

[46] Lemma, E. D., Rizzi, F., Dattoma, T., Spagnolo, B., Sileo, L., Qualtieri, A., De Vittorio, M. and Pisanello, F., "Mechanical properties tunability of three-dimensional polymeric structures in two-photon lithography," IEEE Trans. Nanotechnol. 16(1), 23-31 (2016). 
[47] Juodkazis, S., Mizeikis, V., Seet, K. K., Miwa, M. and Misawa, H., "Two-photon lithography of nanorods in SU8 photoresist," Nanotechnology 16(6), 846-849 (2005).

[48] Baldacchini, T., LaFratta, C. N., Farrer, R. A., Teich, M. C., Saleh, B. E. A., Naughton, M. J. and Fourkas, J. T., "Acrylic-based resin with favorable properties for three-dimensional two-photon polymerization," J. Appl. Phys. 95(11), 6072-6076 (2004).

[49] Xiong, W., Zhou, Y. S., He, X. N., Gao, Y., Mahjouri-Samani, M., Jiang, L., Baldacchini, T. and Lu, Y. F., "Simultaneous additive and subtractive three-dimensional nanofabrication using integrated two-photon polymerization and multiphoton ablation," Light Sci. Appl. 1, e6 (2012).

[50] Gittard, S. D., Nguyen, A., Obata, K., Koroleva, A., Narayan, R. J. and Chichkov, B. N., "Fabrication of microscale medical devices by two-photon polymerization with multiple foci via a spatial light modulator.," Biomed. Opt. Express 2(11), 3167-3178 (2011).

[51] Emons, M., Obata, K., Binhammer, T., Ovsianikov, A., Chichkov, B. N. and Morgner, U., "Two-photon polymerization technique with sub-50 nm resolution by sub-10 fs laser pulses," Opt. Mater. Express 2(7), 942947 (2012).

[52] Madou, M. J., [Fundamentals of microfabrication : the science of miniaturization], CRC Press (2002).

[53] Griesser, H. J., [Thin film coatings for biomaterials and biomedical applications], Elsevier (2016).

[54] Hu, Y., Nieminen, T. A., Heckenberg, N. R. and Rubinsztein-Dunlop, H., "Antireflection coating for improved optical trapping,” J. Appl. Phys. 103(9), 093119 (2008).

[55] Jannasch, A., Demirörs, A. F., Van Oostrum, P. D. J., Van Blaaderen, A. and Schäffer, E., "Nanonewton optical force trap employing anti-reflection coated, high-refractive-index titania microspheres," Nat. Photonics 6, 469473 (2012).

[56] Spesyvtseva, S. E. S. and Dholakia, K., “Trapping in a Material World," ACS Photonics 3(5), 719-736 (2016).

[57] Fischer, M. J. E., "Amine Coupling Through EDC/NHS: A Practical Approach," [Surface Plasmon Resonance], Humana Press, 55-73 (2010).

[58] Valeur, E. and Bradley, M., "Amide bond formation: beyond the myth of coupling reagents," Chem. Soc. Rev. 38(2), 606-631 (2009).

[59] Grace, J. M. and Gerenser, L. J., "Plasma Treatment of Polymers,” J. Dispers. Sci. Technol. 24(3-4), 305-341 (2003).

[60] Shenton, M. J. and Stevens, G. C., "Surface modification of polymer surfaces: atmospheric plasma versus vacuum plasma treatments,” J. Phys. D. Appl. Phys. 34, 2761-2768 (2001).

[61] Hegemann, D., Brunner, H. and Oehr, C., "Plasma Treatment of Polymers to Generate Stable, Hydrophobic Surfaces" (2001).

[62] Dankbar, D. M. and Gauglitz, G., "A study on photolinkers used for biomolecule attachment to polymer surfaces," Anal. Bioanal. Chem. 386(7-8), 1967-1974 (2006).

[63] Blagoi, G., Keller, S., Persson, F., Boisen, A. and Jakobsen, M. H., "Photochemical Modification and Patterning of SU-8 Using Anthraquinone Photolinkers," Langmuir 24(18), 9929-9932 (2008).

[64] Bain, C. D., Troughton, E. B., Tao, Y. T., Evall, J., Whitesides, G. M. and Nuzzo, R. G., "Formation of monolayer films by the spontaneous assembly of organic thiols from solution onto gold," J. Am. Chem. Soc. 111(1), 321-335 (1989).

[65] Bain, C. D. and Whitesides, G. M., "Modeling Organic Surfaces with Self-Assembled Monolayers," Angew. Chemie 28(4), 506-512 (1989). 